\title{
IDENTITIES LINKING VOLUMES OF CONVEX HULLS
}

\author{
RICHARD COWAN, ${ }^{*}$ University of Sydney
}

\begin{abstract}
Let $n$ points be randomly and independently placed in $\mathbb{R}^{d}$ according to a common probability law. It is known that the expected volume for the convex hull of these points, in the cases where $n-d \geq 2$ and even, is related linearly to expected volumes of the convex hulls for $j$ points, $j<n$. We show that similar identities for these volumes hold almost surely — and in contexts where independence and communality of law do not apply. New geometric and topological identities developed here provide a foundation for this result.
\end{abstract}

Keywords: Convex hull; Sylvester's problem; random geometry

2000 Mathematics Subject Classification: Primary 60D05

Secondary $60 \mathrm{C} 05$

\section{Recursive volume formulae}

Consider the random experiment where points $P_{1}, P_{2}, \ldots, P_{n}$ are placed randomly and independently in $\mathbb{R}^{d}(d \geq 1)$ according to a common probability law given by the (induced) probability measure $\mu$ defined on $\mathcal{B}_{d}$, the Borel sets of $\mathbb{R}^{d}$. Let the convex hull of the first $j$ points placed be denoted by $\mathrm{H}_{j}, j=1,2, \ldots, n$. The $d$-dimensional volume measure is denoted by $V$, so the volume of $\mathrm{H}_{j}$ is $V\left(\mathrm{H}_{j}\right)$, which we usually abbreviate to $V_{j}$.

In the important special case of points uniformly distributed on a bounded convex subset $\mathrm{K}$ of $\mathbb{R}^{d}$, our experiment takes the familiar form studied extensively over the last 140 years since the famous four-point problem of Sylvester was first posed. Within this context, and with $d=2$, Affentranger [1] discovered a linear recursive link between $\mathrm{E}\left(V_{n}\right)$ and the expected volumes $\mathrm{E}\left(V_{n-1}\right), \mathrm{E}\left(V_{n-2}\right), \ldots, \mathrm{E}\left(V_{3}\right)$ when $n$ is even. When $d=3$, he proved a similar recursive link for $n$ odd. Using an analytic contribution by Badertscher [4], Affentranger's recursion can be written

$$
\mathrm{E}\left(V_{n}\right)=\sum_{j=1}^{(n-d) / 2}\left(\begin{array}{c}
n \\
2 j-1
\end{array}\right) B_{2 j} \frac{\left(2^{2 j}-1\right)}{j} \mathrm{E}\left(V_{n+1-2 j}\right), \quad(n-d) \geq 2 \text { and even, }
$$

where $B_{r}$ is the $r$ th Bernoulli number. Buchta [6] extended this result by proving (1) for general measure $\mu$ and all dimensions $d$. He also noted the following alternative form of Affentranger's recursion:

$$
\mathrm{E}\left(V_{n}\right)=\frac{1}{2} \sum_{j=1}^{n-d-1}(-1)^{j-1}\left(\begin{array}{l}
n \\
j
\end{array}\right) \mathrm{E}\left(V_{n-j}\right), \quad(n-d) \geq 2 \text { and even. }
$$

Received 13 June 2006; revision received 4 April 2007.

* Postal address: School of Mathematics and Statistics, University of Sydney, NSW 2006, Australia.

Email address: rcowan@mail.usyd.edu.au 
Algebraic manipulation of (2), eliminating the terms where $(n-j-d)$ is even by the sequential use of (2) itself, leads to (1), meaning that the two formulae are closely related.

In this paper we focus on (2), strengthening Buchta's result significantly through the following theorem.

Theorem 1. For any placement method of the points $P_{1}, P_{2}, \ldots, P_{n}$ in $\mathbb{R}^{d}$, either random or not,

$$
V_{n}=\frac{1}{2} \sum_{j=1}^{n-d-1}(-1)^{j-1} \sum_{s \in \S_{n-j}} V(\mathrm{H}(s)), \quad(n-d) \geq 2 \text { and even, }
$$

where $s_{j}$ is the set of $j$-subsets of the points $P_{1}, P_{2}, \ldots, P_{n}$ and, for $s \in s_{j}, \mathrm{H}(s)$ is defined as the convex hull of $s$. We can write this as

$$
V_{n}=\frac{1}{2} \sum_{j=1}^{n-d-1}(-1)^{j-1}\left(\begin{array}{l}
n \\
j
\end{array}\right) \bar{V}_{n-j}^{(n)}, \quad(n-d) \geq 2 \text { and even, }
$$

where $\bar{V}_{j}^{(n)}$ is defined as the average volumes for all $\left(\begin{array}{c}n \\ j\end{array}\right) j$-hulls of $P_{1}, P_{2}, \ldots, P_{n}$. So Buchta's formula, (2), holds in any random context where $\mathrm{E}\left(\bar{V}_{j}^{(n)}\right)=\mathrm{E}\left(V_{j}\right)$ for all $j<n$.

This purely geometric result adds considerable insight to the random situation described above, whilst also facilitating analyses of random-geometric applications where independence and/or communality of distribution have been dropped.

\section{Applications of a wider nature}

We can now deal, for example, with the situation where $n$ points are placed exchangeably. By this we mean that a probability measure, $\mu_{n}$ say, on $\left(\mathbb{R}^{d}\right)^{n}$ endowed with the usual $\sigma$-algebra generated by product Borel sets, has the following property: $\mu_{n}\left(D_{1} \times D_{2} \times \cdots \times D_{n}\right)=$ $\mu_{n}\left(D_{\rho(1)} \times D_{\rho(2)} \times \cdots \times D_{\rho(n)}\right)$ for any $D_{1}, D_{2}, \ldots, D_{n} \in \mathscr{B}_{d}$ and any permutation $\rho$. We retain the notation $\mu$ as $\mu(D):=\mu_{n}\left(D \times \mathbb{R}^{d} \times \cdots \times \mathbb{R}^{d}\right)$ for all $D \in \mathcal{B}_{d}$. Exchangeability of the first $n$ placements means that the first $j$ points are placed exchangeably for all $2 \leq j<n$.

Importantly, exchangeability for the locations of $P_{1}, P_{2}, \ldots, P_{n}$ implies that the volumes of all $\left(\begin{array}{l}n \\ j\end{array}\right)$ convex hulls derived from $j$-subsets of the points $P_{1}, P_{2}, \ldots, P_{n}$ are identically distributed, with each distributed as $V\left(\mathrm{H}_{j}\right)$ and having common expectation $\mathrm{E}\left(V_{j}\right)$. So $\mathrm{E}\left(\bar{V}_{j}^{(n)}\right)=$ $\mathrm{E}\left(V_{j}\right)$, satisfying the condition, stated in Theorem 1, for (2) to hold.

Example 1. Let $\left\{Q_{1}, Q_{2}, \ldots, Q_{m}\right\}, m \geq n$, be an arbitrary set of points in $\mathbb{R}^{d}$-we call it the base layout. Sample $P_{i}, i=1,2, \ldots, n$, points randomly without replacement from this set (with uniform distribution from those still available for selection). The $P_{i}$ are exchangeable and $\mu$ has probability measure $1 / m$ at each point in the base layout.

Example 2. Let $m=7$ in Example 1, with six of the points $Q_{i}$ being placed at the vertices of a regular hexagon in $\mathbb{R}^{2}$ (of unit area) and the seventh in the hexagon's centre. When $n=6$, we can see that $\mathrm{H}_{6}$ is a random set. With probability $\frac{1}{7}$ it is the regular hexagon; it is a pentagon of area $\frac{5}{6}$ with probability $\frac{6}{7}$.

All of the column headings in Table 1 are random variables, being functionals of the random set $\mathrm{H}_{6}$, but we see that (4) holds for each random version of $\mathrm{H}_{6}$. Formula (2) also holds. 
TABLE 1.

\begin{tabular}{cccccccc}
\hline & $\bar{V}_{1}^{(6)}$ & $\bar{V}_{2}^{(6)}$ & $\bar{V}_{3}^{(6)}$ & $\bar{V}_{4}^{(6)}$ & $\bar{V}_{5}^{(6)}$ & $\sum_{i}(-1)^{i-1}\left(\begin{array}{c}n \\
i\end{array}\right) \bar{V}_{i}^{(6)}$ & $2 V_{6}$ \\
\hline Hexagon & 0 & 0 & $\frac{6}{20}$ & $\frac{9}{15}$ & $\frac{5}{6}$ & 2 & 2 \\
Pentagon & 0 & 0 & $\frac{13}{60}$ & $\frac{13}{30}$ & $\frac{23}{36}$ & $\frac{5}{3}$ & $\frac{5}{3}$ \\
\hline
\end{tabular}

Example 3. The $n$ points might be placed exchangeably using Strauss' model [9]—or one of the more elaborate 'interacting-points' models that have developed from it; for example, area-interaction models [3] and nearest-neighbour Markov models [2].

Example 4. The points $P_{1}, P_{2}, \ldots, P_{n}$ are placed sequentially in $\mathbb{R}^{d}$ according to independent sampling of $\mu$, except that the $j$ th point $(j \geq 2)$ must be resampled until its location is not within a distance $r$ of $P_{j-1}$. This sequential Markovian dependence does not create exchangeability.

Other point-construction methods which illustrate the widened repertoire of applications are easily imagined.

Example 5. Place $I$ points randomly within the subset $A \subset \mathbb{R}^{d}$ according to measure $\mu_{A}$, and $J$ randomly within the subset $B \subset \mathbb{R}^{d}$ according to $\mu_{B}, I+J=n$. For example, let $A$ be the interior of a set K and $B$ be K's boundary. Miles [8] studied this case when $\mathrm{K}$ is a ball and both measures are uniform within their domains.

Example 6. The points can be constructed from more elaborate random-geometric objects. For example, let $\mathrm{K}$ be a bounded convex set in $\mathbb{R}^{2}$ and draw $k$ isotropic uniformly random secants. The intersection of these secants with the boundary of $\mathrm{K}$ create $n=2 k$ points and hence a hull, $\mathrm{H}_{2 k}$. The secant-secant intersections within $\mathrm{K}$ constitute another collection of points, but as their number is random, not fixed in advance, they do not fit our theory.

\section{A topological identity for convex hulls}

Behind Theorem 1 lies a beautiful identity of a topological character. Place $n$ points $P_{1}, P_{2}, \ldots, P_{n}, n \geq 1$ in $\mathbb{R}^{d}$. The locations of these points are arbitrary; we allow points to be collinear, coplanar, or coincident with each other. We even permit the convex hull $\mathrm{H}_{n}$ of all $n$ points to lie in a flat of dimension less than $d$, an arrangement that we call completely aligned. To take account of such an alignment, however, we introduce the dimension of $\mathrm{H}_{n}$ and denote it by $h$. Effectively the action takes place in dimension $h$, so if $h<d$ we tacitly identify the $h$-flat in $\mathbb{R}^{d}$ which contains $\mathrm{H}_{n}$ with the space $\mathbb{R}^{h}$. Naturally $1 \leq h \leq \min (d, n-1)$.

Later in the paper, when we discuss the random context, the experiment is set in $\mathbb{R}^{d}$ but the arrangement of points may, by chance, have lower-dimensional convex hull; then $h$ is a random variable with range $1 \leq h \leq \min (d, n-1)$. The distinction between $d$ and $h$ is a necessary one.

If $n>d$, there may be no examples of $j$ points being contained in a $(j-2)$-flat (for any $j \leq n$ ); the placement of points is then called completely unaligned (the usual geometer's phrase 'in general position' being unsuitable for an arrangement which is less general than the 'arbitrary' premises just stated).

Theorem 2. $P_{1}, P_{2}, \ldots, P_{n}, n \geq 1$, are points in $\mathbb{R}^{d}$ whose convex hull has dimension $h \leq$ $\min (d, n-1)$. For any reference point $P \in \mathrm{H}_{n}$, define $c_{j}(P)$ as the number of sub-collections 


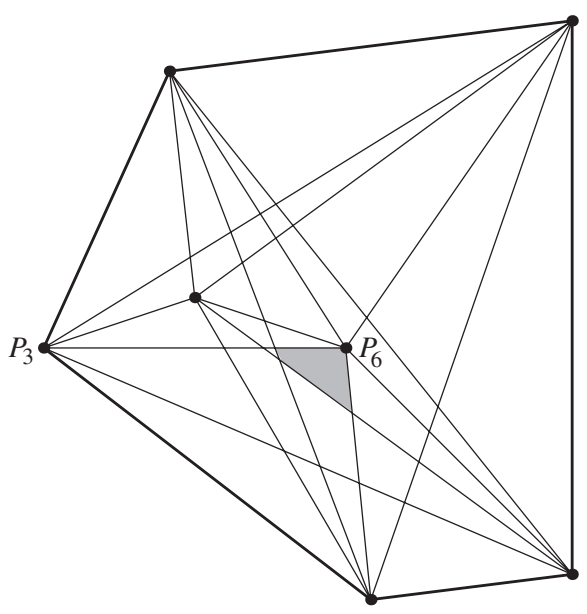

(a)

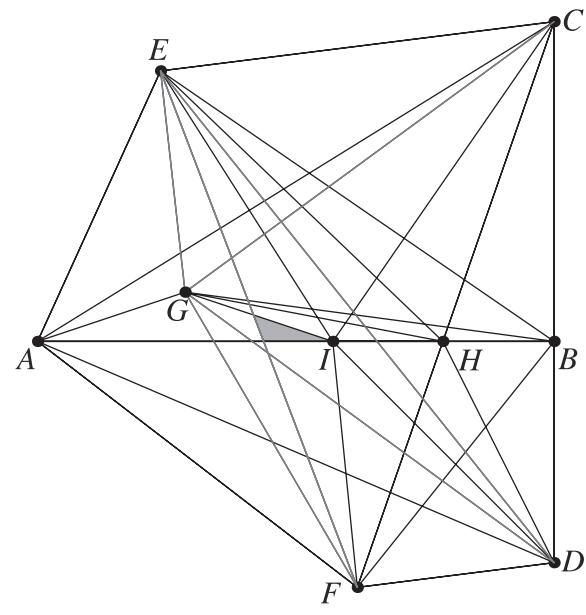

(b)

FigURE 1: (a) A completely unaligned layout of seven points in $\mathbb{R}^{2}$. So $h=d=2 . \mathrm{H}_{7}$ is shown along with all the 2-hulls, one of which (derived from $P_{3}$ and $P_{6}$ ) is marked. (b) Some collinearities exist, so this layout is not completely unaligned. Once again $h=d=2$. Suppose also that there are coincident points at some of the nine black dots $A-H$, say three at $G$ and two at $B$. Therefore, $n=12$.

of $j$ points taken from $\left\{P_{1}, P_{2}, \ldots, P_{n}\right\}$ whose convex hull contains $P$. Then,

$$
\begin{aligned}
\Psi(P):=c_{1}(P)-c_{2}(P)+\cdots+(-1)^{n-1} c_{n}(P) & =(-1)^{h} & & \text { for almost all } P \in \stackrel{\circ}{\mathrm{H}}_{n}, \\
& =0 & & P \in \partial \mathrm{H}_{n},
\end{aligned}
$$

where $\partial \mathrm{H}_{n}$ and $\stackrel{\circ}{\mathrm{H}}_{n}$ respectively denote the boundary and interior of $\mathrm{H}_{n}$, treated for topological purposes as a set in the identified space $\mathbb{R}^{h}$. Trivially, $\Psi(P)=0$ if we consider $P \notin \mathrm{H}_{n}$.

Remark 1. The case in which $h=0$ is covered by Theorem 2. Recall that the interior and boundary of $\mathbb{R}^{0}$ are equal to $\mathbb{R}^{0}$ and $\varnothing$, respectively. Also, $d=0$ is permitted. The simple form of the theorem hides considerable counting complexity. As an exercise, suppose that $P$ is placed in the interior of the shaded zone of Figure $1(\mathrm{a})$. Then $\Psi(P)=0-0+12-26+21-7+1=1$. If $P$ is located at $H$ in Figure $1(b)$, then $\Psi(P)=1-16+114-\cdots+782-494+\left(\begin{array}{c}12 \\ 9\end{array}\right)-$ $\left(\begin{array}{l}12 \\ 10\end{array}\right)+\left(\begin{array}{l}12 \\ 11\end{array}\right)-1$, but filling in the three missing terms, $c_{4}(P), c_{5}(P)$, and $c_{6}(P)$, is a challenge to human counting skills.

I have not found this topological identity, which has a superficial appearance reminiscent of functionals which appear in Euler's formula or in the definitions of the Euler Characteristic, within the topological literature. It has apparently been overlooked, perhaps because a mathematical structure composed of these many convex hulls does not fit naturally into the framework of CW-complexes, the versatile and commonly studied cellular system of modern topology. In the random-geometry literature the identity has not been recognised either, although the trivial case in which $n=h+2$ and $h=d$ is stated as a lemma by Miles [8, p. 372] and used implicitly by Buchta in [5].

Clearly, Theorem 2 (which we prove in Section 4) has the following corollary, which in turn proves Theorem 1 . 
Corollary 1. Let $v$ be a $\sigma$-finite measure on $\left(\mathbb{R}^{h}, \mathscr{B}_{h}\right)$. For $1 \leq j \leq n$, define $\bar{v}_{j}^{(n)}$ as the average of $v$ measures over the $\left(\begin{array}{c}n \\ j\end{array}\right)$ convex hulls formed from all $j$-subsets of $P_{1}, P_{2}, \ldots, P_{n}$. Then

$$
\begin{aligned}
\sum_{j=1}^{n-1}(-1)^{j-1} \bar{v}_{n-j}^{(n)} & =2 \bar{v}\left(\stackrel{\circ}{\mathrm{H}}_{n}\right)+\bar{v}\left(\partial \mathrm{H}_{n}\right), & & n-h \geq 2 \text { and even }, \\
& =\bar{v}\left(\partial \mathrm{H}_{n}\right), & & n-h \geq 3 \text { and odd } .
\end{aligned}
$$

When $v$ is absolutely continuous with respect to the h-dimensional Lebesgue measure, we have

$$
\begin{aligned}
\frac{1}{2} \sum_{j=1}^{n-h-1}(-1)^{j-1} \bar{v}_{n-j}^{(n)} & =v\left(\mathrm{H}_{n}\right) \equiv \bar{v}_{n}^{(n)}, & & n-h \geq 2 \text { and even } \\
& =0, & & n-h \geq 3 \text { and odd } .
\end{aligned}
$$

Proof. For $P \in \mathbb{R}^{h}$ and $\mathrm{H} \subset \mathbb{R}^{h}$ let $\mathbf{1}_{\mathrm{H}}(\cdot)$ be defined as the indicator function of the domain $\mathrm{H}$, namely $\mathbf{1}_{\mathrm{H}}(P)=1$ if $P \in \mathrm{H}$ and 0 otherwise. Clearly,

$$
\int_{\mathbb{R}^{h}} \mathbf{1}_{\mathrm{H}}(P) v(\mathrm{~d} P)=v(\mathrm{H}) .
$$

The entity $c_{j}$ in Theorem 2 is the sum of the indicator functions of the $j$-subset convex hulls. So,

$$
\int_{\mathbb{R}^{h}} c_{j}(P) v(\mathrm{~d} P)=\left(\begin{array}{c}
n \\
j
\end{array}\right) \bar{v}_{j}^{(n)} .
$$

Therefore, (5) and (6) follow from an integration of the identity in Theorem 2. With $n \geq h+1$, we have the following:

$$
\int_{\mathbb{R}^{h}} \Psi(P) v(\mathrm{~d} P)=\int_{\mathrm{H}_{n}}(-1)^{h} v(\mathrm{~d} P) .
$$

Therefore, $\left(\begin{array}{c}n \\ 1\end{array}\right) \bar{\nu}_{1}^{(n)}-\left(\begin{array}{c}n \\ 2\end{array}\right) \bar{\nu}_{2}^{(n)}+\cdots+(-1)^{n-1}\left(\begin{array}{l}n \\ n\end{array}\right) \bar{\nu}_{n}^{(n)}=(-1)^{h} v\left(\stackrel{\circ}{\mathrm{H}}_{n}\right)$, and so,

$$
\begin{aligned}
\sum_{j=1}^{n-1}(-1)^{j-1}\left(\begin{array}{l}
n \\
i
\end{array}\right) \bar{v}_{j}^{(n)}+(-1)^{n-1} v\left(\partial \mathrm{H}_{n}\right) & =\left((-1)^{h}-(-1)^{n-1}\right) v\left(\stackrel{\circ}{\mathrm{H}}_{n}\right) \\
& =2(-1)^{n} v\left(\stackrel{\circ}{\mathrm{H}}_{n}\right) \quad \text { when }(n-h) \text { is even. }
\end{aligned}
$$

Therefore,

$$
\begin{array}{rlrl}
\sum_{j=1}^{n-1}(-1)^{j-1}\left(\begin{array}{l}
n \\
i
\end{array}\right) \bar{v}_{n-j}^{(n)} & =2 v\left(\stackrel{\circ}{\mathrm{H}}_{n}\right)+v\left(\partial \mathrm{H}_{n}\right) & & \text { when }(n-h) \text { is even, } \\
& =v\left(\partial \mathrm{H}_{n}\right) & \text { when }(n-h) \text { is odd. }
\end{array}
$$

We have used the obvious fact that $\bar{v}_{n}^{(n)}=v\left(\mathrm{H}_{n}\right)$. Identity (7) follows as an example of (5), in which absolute continuity allows us to ignore $v\left(\partial \mathrm{H}_{n}\right)$ and also to set $\bar{v}_{j}^{(n)}=0$ for all $j \leq h$. 
Remark 2. Mainly we shall use (5) and (7) in the sequel. It turns out that (6) and (8) are somewhat redundant to our needs because (5) implies (6) and (7) implies (8).

The first use of (7) is, of course, the immediate proof of Theorem 1 by setting $v$ equal to the $d$-dimensional measure $V$ (restricted to the $h$-dimensional flat which contains $\mathrm{H}_{n}$, if $h<d$ ).

Proof of Theorem 1. If $h=d$, then (3) follows immediately from (7). The elimination of summation terms, $(n-d) \leq j \leq(n-1)$, follows because $\ell_{n-j}$ contains only sets of dimension less than $d$ for that range of $j$. If $h<d$, then $V_{n}$ and each $V(\mathrm{H}(s))$ in (3) equal 0 , so (3) is true in a degenerate way. Thus, (3) is true for all positions of $P_{1}, P_{2}, \ldots, P_{n}$ regardless of the variable $h$. Formula (4) and the last remark of Theorem 1 follow trivially.

In the random setting, we define $\eta_{x}:=\mathrm{P}\{h=x\}, 0 \leq x \leq d$. Sometimes $\eta_{j}$ can be calculated easily; for example, in the seven-point base layout used in Example 2, $\eta_{2}=1$ for $4 \leq n \leq 7$, but if $n=3$ then $\eta_{1}=\frac{3}{35}$ and $\eta_{2}=\frac{32}{35}$.

Example 7. In $\mathbb{R}^{2}$, consider independent placement with $\mu$ totally concentrated on three points whose convex hull is a triangle with area $a>0$. The probability weights are $p_{1}, p_{2}$, and $p_{3}$. It is readily shown that $V_{n}=a$ with probability

$$
1+p_{1}^{n}+p_{2}^{n}+p_{3}^{n}-\left(1-p_{1}\right)^{n}-\left(1-p_{2}\right)^{n}-\left(1-p_{3}\right)^{n}
$$

(an entity which is 0 for $n<3$ ), and $V_{n}=0$ otherwise. So $\eta_{0}=p_{1}^{n}+p_{2}^{n}+p_{3}^{n}$ and

$$
\eta_{1}=\left(1-p_{1}\right)^{n}+\left(1-p_{2}\right)^{n}+\left(1-p_{3}\right)^{n}-2 p_{1}^{n}-2 p_{2}^{n}-2 p_{3}^{n},
$$

whilst $\eta_{2}=\mathrm{P}\left\{V_{n}=a\right\}$.

Other elementary calculations confirm Buchta's identity, (2). The right-hand side of (2), when $n \geq 4$ and even, equals

$$
\begin{aligned}
\frac{a}{2} \sum_{j=1}^{n-3}(-1)^{j-1}\left(\begin{array}{c}
n \\
j
\end{array}\right)\left[1+\sum_{i=1}^{3} p_{i}^{n-j}-\sum_{i=1}^{3}\left(1-p_{i}\right)^{n-j}\right] \\
=\frac{a}{2}\left[\left(2+\frac{1}{2} n(n-1)-n-(1-1)^{n}\right)\right. \\
+\sum_{i=1}^{3}\left(1+p_{i}^{n}+\frac{1}{2} n(n-1) p_{i}^{2}-n p_{i}-\left(1-p_{i}\right)^{n}\right) \\
\left.-\sum_{i=1}^{3}\left(1+\left(1-p_{i}\right)^{n}+\frac{1}{2} n(n-1)\left(1-p_{i}\right)^{2}-n\left(1-p_{i}\right)-p_{i}^{n}\right)\right] \\
=\frac{a}{2}\left[2-n(n-1)+2 n+2 \sum_{i=1}^{3}\left(p_{i}^{n}+\frac{1}{2} n(n-1) p_{i}-n p_{i}-\left(1-p_{i}\right)^{n}\right)\right] \\
=a\left[1+\sum_{i=1}^{3}\left(p_{i}^{n}-\left(1-p_{i}\right)^{n}\right)\right] \\
=\operatorname{left-hand~side.~}
\end{aligned}
$$




\section{Proof of Theorem 2}

We turn to the proof of Theorem 2, but before commencing formalities, we discuss the basic geometry of convex hulls (aided by visual assistance when $d=2,3$ from Figures 1 and 2, respectively).

- $\mathrm{H}_{n}$ is an $h$-polytope, with faces of dimensions $0,1, \ldots,(h-1)$ on its boundary. In general, its facets, that is the $(h-1)$-faces, are $(h-1)$-polytopes. In the completely unaligned case, however, these facets are $(h-1)$-simplices as there will be no occurrence of more than $h$ points lying in any $(h-1)$-flat. By contrast, note in Figure 1(b) the four points lying in the 1-flat which contains $C D$, one of the sides of $\mathrm{H}_{n}$.

- Recall that an $h$-object is an object of $h$-dimensions (where 'object' can be polytope, flat, simplex, face, etc.); see [7]. One exception in this paper is the $j$-hull which is generated from $j \geq 1$ points and may be an object of any dimension less than or equal to $\min (h, j-1)$ if the points are in $\mathbb{R}^{h}$.

- Let $U$ be defined as the union of all $h$-hulls. Here $U$, seen as a network of line-segments in Figure 1, partitions $\mathrm{H}_{n}$ into a tessellation. That is, $\mathrm{H}_{n} \backslash U$ is a collection of disjoint, open, connected subsets (called 'zones') whose closures cover $\mathrm{H}_{n}$. Each zone $Z$ is the interior of an $h$-polytope. One such zone is shaded in each part of Figure 1. We define an $i$-face of a zone $Z$ as the interior of the corresponding $i$-face of $Z$ 's closure; this is an open set when $i>0$.

- Let $Z$ be a zone. For all $P \in Z, c_{j}(P)$ is a constant; in particular, $c_{j}(P)=0$ for $1 \leq j \leq h, P \in Z$.

- Figure 2(a) shows the polyhedron (3-polytope) $\mathrm{H}_{7}$ which arose from seven uniformly random points inside the unit cube. Only three facets are seen from the viewing point. When the two most prominent facets and all structure above the line $z=\frac{2}{5}$ are removed (as in Figure 2(b)), we see some of the architecture of $U$. In Figure 2(c), the union of two hulls is shown as a 'net' of $\left(\begin{array}{l}7 \\ 2\end{array}\right)$ line segments in $\mathbb{R}^{3}$. Some edges (1-faces) of zones

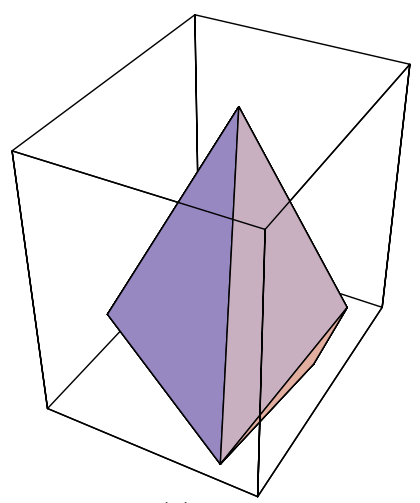

(a)

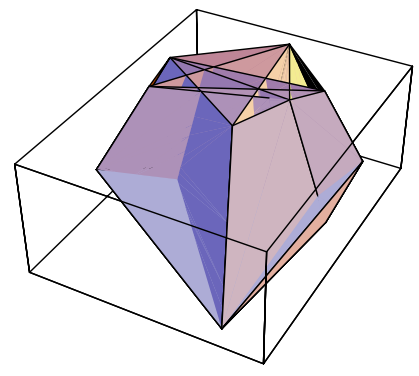

(b)

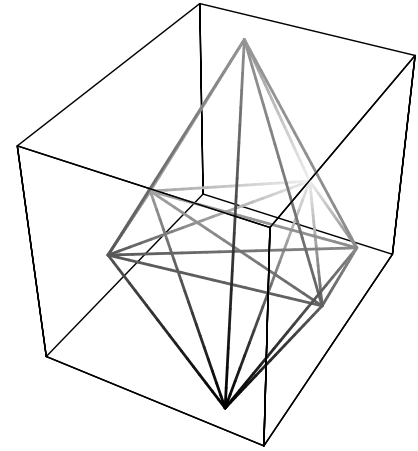

(c)

Figure 2: (a) A view of one realisation of $\mathrm{H}_{7}$ in $\mathbb{R}^{3}$, with only three facets visible. (b) Two of these three facets have been removed, as has all structure above $z=\frac{2}{5}$, rendering some of the 3-hulls more visible.

(c) The net of 2-hulls, with blacker shading indicating closeness to the viewing point. 


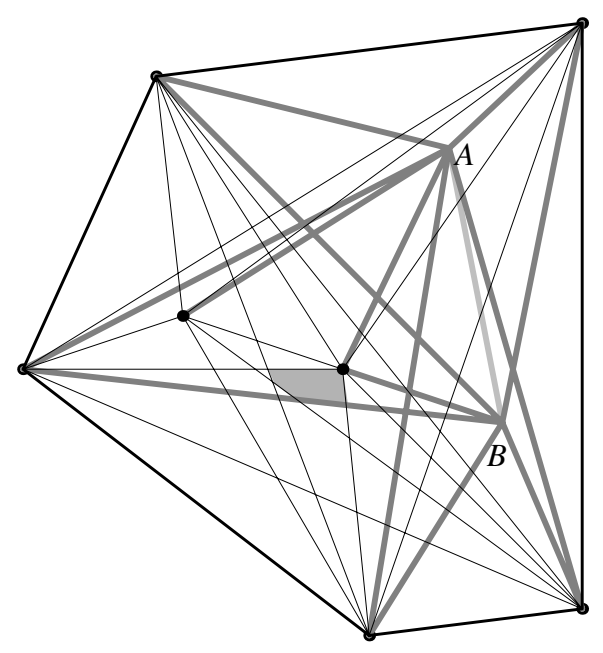

Figure 3: Here $n=10$, with seven points lying on the 2-flat in which this page of the journal lies and the three others in $\mathbb{R}^{3}$, not on the page. The thick grey lines are the intersection of various 3-hulls with the page. See text for further detail.

lie in this net, but some do not (just as in Figure 1, some of the 0 -faces of zones lie in the set of 1-hulls but some, indeed most, do not).

- In the completely unaligned case, any $h$-hull is an $(h-1)$-simplex. The $(h-1)$-flat containing this $h$-hull does not contain any $P_{i}$ other than the $h$ which generated the hull. In general, there may be many $h$-hulls lying in one $(h-1)$-flat. For example, in Figure 1(b) the line (1-flat) containing $A B$ contains $\left(\begin{array}{l}f \\ 2\end{array}\right)$ 2-hulls, where $f$ equals the number of points on that flat (i.e. $f=c_{1}(A)+c_{1}(I)+c_{1}(H)+c_{1}(B)=5$ ). For a more complicated example, consider that the seven points in Figure 1(a) lie on a common 2-flat in $\mathbb{R}^{3}$. There are $\left(\begin{array}{l}7 \\ 3\end{array}\right)$ 3-hulls lying within that flat. Their union is the convex hull of the seven points. Note, however, that the structure on this 2-flat is further complicated by the other 3-hulls constructed using points which do not lie on the flat. The thick grey lines in Figure 3 illustrate this complication for the case in which $n=10$, where there are two points ( $P_{5}$ and $P_{9}$, say) above the flat, one point $\left(P_{2}\right.$, say) below the flat, and the remaining seven points on the flat. $A$ and $B$ are the points where the 2-hulls $P_{2} P_{5}$ and $P_{2} P_{9}$ intersect the flat.

- More formally, the relationship 'lies in a common $(h-1)$-flat' is an equivalence relationship on the set of $h$-hulls. So this set can be partitioned into equivalence classes. Now $U$ can be represented as

$$
\bigcup_{\mathrm{F} \in \mathcal{F}_{h}} \operatorname{conv}\left(P_{i}: P_{i} \in \mathrm{F}\right),
$$

where $\mathcal{F}_{k}$ is the class of all $(h-1)$-flats which contain at least $k$ points from $\left\{P_{1}, P_{2}, \ldots, P_{n}\right\}$. Thus, in Figure 1(b), $U$, which is defined as the union of all 2-hulls, can be represented as $A B \cup F C \cup C D \cup\{24$ other letter pairs $\}$, a saving on the $\left(\begin{array}{c}12 \\ 2\end{array}\right)=66$ point-pairs.

The following lemma captures one of the essential notions in the proof of Theorem 2. 
Lemma 1. With the action taking place in $h \geq 1$ dimensions, let exactly $f$ points from $\left\{P_{1}, P_{2}, \ldots, P_{n}\right\}$ lie on some $(h-1)$-flat called $\mathrm{F}$. Here $0 \leq f<n$. The flat $\mathrm{F}$ divides the space $\mathbb{R}^{h}$ into two open half-spaces, called $\mathrm{F}^{+}$and $\mathrm{F}^{-}$say. Denote the numbers of points from $\left\{P_{1}, P_{2}, \ldots, P_{n}\right\}$ lying in $\mathrm{F}^{+}$or in $\mathrm{F}^{-}$by $n^{+}$or $n^{-}$, respectively. Here $f+n^{+}+n^{-}=n$. For a reference point $P \in \mathrm{F}$, define

$$
\begin{aligned}
c_{j}^{+}(P):= & \text { number of } j \text {-hulls covering } P \text { and intersecting } \mathrm{F}^{+} \text {but not } \mathrm{F}^{-}, \\
c_{j}^{-}(P):= & \text { number of } j \text {-hulls covering } P \text { and intersecting } \mathrm{F}^{-} \text {but not } \mathrm{F}^{+}, \\
c_{j}^{ \pm}(P):= & \text { number of } j \text {-hulls covering } P \text { and intersecting both } \mathrm{F}^{+} \text {and } \mathrm{F}^{-}, \\
c_{j}^{\varnothing}(P):= & \text { number of } j \text {-hulls covering } P \text { and intersecting neither } \mathrm{F}^{+} \text {or } \mathrm{F}^{-} \\
& \left(\text {i.e. hull } \cap\left(\mathrm{F}^{+} \cup \mathrm{F}^{-}\right)=\varnothing\right) .
\end{aligned}
$$

If $\Psi^{\star}(P):=\sum_{j=1}^{n}(-1)^{j-1} c_{j}^{\star}(P)$ for any symbol $\star$, then

$$
\begin{aligned}
\Psi^{+}(P) & =-\Psi^{\varnothing}(P) & & \text { provided } n^{+}>0, \\
\Psi^{-}(P) & =-\Psi^{\varnothing}(P) & & \text { provided } n^{-}>0, \\
\Psi(P) & =\Psi^{ \pm}(P)-\Psi^{\varnothing}(P) & & \text { provided } n^{+}>0 \text { and } n^{-}>0 .
\end{aligned}
$$

Also $\Psi^{+}(P)=0$ if $n^{+}=0, \Psi^{-}(P)=0$ if $n^{-}=0$, and $\Psi(P)=0$ if $\min \left(n^{+}, n^{-}\right)=0$.

Proof. Obviously

$$
c_{j}(P)=c_{j}^{\varnothing}(P)+c_{j}^{+}(P)+c_{j}^{-}(P)+c_{j}^{ \pm}(P)
$$

and, so, we address the terms on the right-hand side, $c_{j}^{+}(P)$, first. By combining selections of $j$ points, some in $\mathrm{F}^{+}$and the rest in $\mathrm{F}$ with convex hull covering $P$, we obtain, for $2 \leq j \leq n^{+}+f$,

$$
\begin{aligned}
c_{j}^{+}(P) & =\left(\begin{array}{c}
n^{+} \\
1
\end{array}\right)\left(\begin{array}{c}
c_{j-1}^{\varnothing}(P) \\
1
\end{array}\right)+\left(\begin{array}{c}
n^{+} \\
2
\end{array}\right)\left(\begin{array}{c}
c_{j-2}^{\varnothing}(P) \\
1
\end{array}\right)+\cdots+\left(\begin{array}{c}
n^{+} \\
n^{+}
\end{array}\right)\left(\begin{array}{c}
c_{j-n^{+}}^{\varnothing}(P) \\
1
\end{array}\right) \\
& =\sum_{i=1}^{n^{+}}\left(\begin{array}{c}
n^{+} \\
i
\end{array}\right) c_{j-i}^{\varnothing}(P),
\end{aligned}
$$

whilst $c_{j}^{+}(P)=0$ when $j=1$ or $j>n^{+}+f$. There might be 0 terms in this expression, because $c_{j-i}^{\varnothing}(P)=0$ if $j-i>f$ or $j-i \leq 0$. Therefore,

$$
\begin{aligned}
& \Psi^{+}(P)=\sum_{j=2}^{n^{+}+f}(-1)^{j-1} c_{j}^{+}(P) \\
& \text { since } c_{j}^{+}(P)=0 \text { when } j>n^{+}+f \\
& =\sum_{j=2}^{n^{+}+f}(-1)^{j-1} \sum_{i=1}^{n^{+}}\left(\begin{array}{c}
n^{+} \\
i
\end{array}\right) c_{j-i}^{\varnothing}(P) \\
& =\sum_{i=1}^{n^{+}}\left(\begin{array}{c}
n^{+} \\
i
\end{array}\right) \sum_{j=2}^{n^{+}+f}(-1)^{j-1} c_{j-i}^{\varnothing}(P) \\
& =\sum_{i=1}^{n^{+}}\left(\begin{array}{c}
n^{+} \\
i
\end{array}\right) \sum_{j=i+1}^{n^{+}+f}(-1)^{j-1} c_{j-i}^{\varnothing}(P) \\
& \text { since } c_{j-i}^{\varnothing}(P)=0 \text { when } j<i+1
\end{aligned}
$$




$$
\begin{aligned}
& =\sum_{i=1}^{n^{+}}\left(\begin{array}{c}
n^{+} \\
i
\end{array}\right)(-1)^{i} \sum_{t=1}^{n^{+}+f-i}(-1)^{t-1} c_{t}^{\varnothing}(P) \quad \text { where } t:=j-i \\
& =\sum_{i=1}^{n^{+}}\left(\begin{array}{c}
n^{+} \\
i
\end{array}\right)(-1)^{i} \sum_{t=1}^{f}(-1)^{t-1} c_{t}^{\varnothing}(P) \quad \text { since } c_{t}^{\varnothing}(P)=0 \text { when } t>f \\
& =\left((1-1)^{n^{+}}-1\right) \Psi^{\varnothing}(P) \\
& =-\Psi^{\varnothing}(P), \quad n^{+}>0 \text {. }
\end{aligned}
$$

Likewise, $\Psi^{-}(P)=-\Psi^{\varnothing}(P)$ provided $n^{-}>0$, so, we have established (9) and (10). From these new findings and (12), with $\min \left(n^{+}, n^{-}\right)>0$, we have

$$
\begin{aligned}
\Psi(P) & =\Psi^{\varnothing}(P)+\Psi^{+}(P)+\Psi^{-}(P)+\Psi^{ \pm}(P) \\
& =\Psi^{\varnothing}(P)-\Psi^{\varnothing}(P)-\Psi^{\varnothing}(P)+\Psi^{ \pm}(P) \\
& =\Psi^{ \pm}(P)-\Psi^{\varnothing}(P),
\end{aligned}
$$

confirming (11). When $n^{+}=0, \Psi^{+}(P)=\Psi^{ \pm}(P)=0, \Psi^{-}(P)=-\Psi^{\varnothing}(P)$, so $\Psi(P)=0$ (and likewise if $\left.n^{-}=0\right)$. Finally we note that, if $f=0$, the arguments and conclusions above are valid; also, $\Psi^{\varnothing}(P)=0$, reducing (9)-(11) to obvious truths.

Remark 3. There is little prospect of finding an expression for $c_{j}^{ \pm}(P)$ for general $h, f, n^{+}$, and $n^{-}$and general point-locations. $P$-covering $j$-hulls which intersect both $\mathrm{F}^{+}$and $\mathrm{F}^{-}$include those where

(a) none of the $j$ points lie in $\mathrm{F}$ or

(b) $P$ is not in the convex hull of those that do lie in F.

In Figure 1(b), examples of (a) based on $\mathrm{F} \supset A B$ and $P \in \mathrm{F}$ in the neighbourhood of $I$ are: 3-hulls $E D F, E C F, G C F$, and $E D G$; 4-hulls $E C F G$ and $E D F G$. Examples of (b) for the same F and $P$ are: 3-hulls $E D A, E H F$, and $E B F$; 4-hulls $E B F H, G H D F$, and $G B F H$. Multiplicities in these lists also occur because of the coincidences at $B$ and $G$. In general, these types of $P$-coverings are very difficult to count.

Lemma 2. Using the premises of Lemma 1, define $f_{j}(\mathrm{~F})$ as the number of $j$-hulls which intersect with the flat $\mathrm{F}$. Then

$$
f_{j}(\mathrm{~F})=\left(\begin{array}{c}
n \\
j
\end{array}\right)-\left(\begin{array}{c}
n^{+} \\
j
\end{array}\right)-\left(\begin{array}{c}
n^{-} \\
j
\end{array}\right) .
$$

Proof. This trivial expression is the total number of $j$-hulls minus those that do not intersect $\mathrm{F}$.

Proof of Theorem 2 for $h \leq 1$. The case in which $h=0$ is trivial, so we consider only $h=1$. For all $P$ and $j, c_{j}(P)=f_{j}(\mathrm{~F})$, based on a 0 -flat $\mathrm{F}$ located at $P$. This equals $\left(\begin{array}{c}n \\ j\end{array}\right)-\left(\begin{array}{c}n^{+} \\ j\end{array}\right)-\left(\begin{array}{c}n^{-} \\ j\end{array}\right)$, from (13). Alternatively, a derivation of this comes from

$$
c_{j}(P)=c_{j}^{+}(P)+c_{j}^{-}(P)+c_{j}^{ \pm}(P)+c_{j}^{\varnothing}(P),
$$




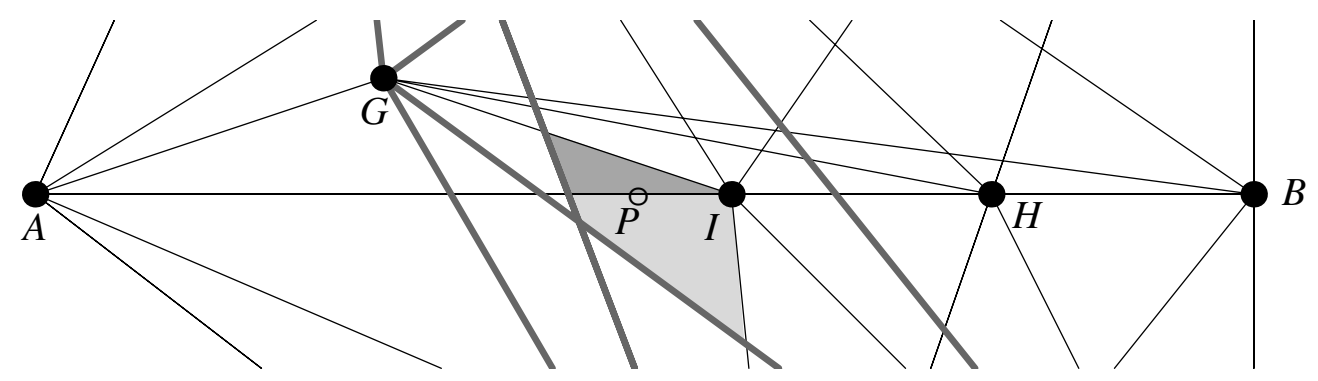

FIGURE 4: This drawing, which is an enlargement of part of Figure 1(b), illustrates Lemma 3.

where all terms, including $c_{j}^{ \pm}(P)$, are easily derived when $h=1$,

$$
\begin{aligned}
& c_{j}^{+}(P)=\left(\begin{array}{c}
n^{+}+f \\
j
\end{array}\right)-\left(\begin{array}{c}
n^{+} \\
j
\end{array}\right)-\left(\begin{array}{l}
f \\
j
\end{array}\right), \\
& c_{j}^{-}(P)=\left(\begin{array}{c}
n^{-}+f \\
j
\end{array}\right)-\left(\begin{array}{c}
n^{-} \\
j
\end{array}\right)-\left(\begin{array}{l}
f \\
j
\end{array}\right), \\
& c_{j}^{ \pm}(P)=\left(\begin{array}{c}
n \\
j
\end{array}\right)-\left(\begin{array}{c}
n^{+}+f \\
j
\end{array}\right)-\left(\begin{array}{c}
n^{-}+f \\
j
\end{array}\right)+\left(\begin{array}{l}
f \\
j
\end{array}\right), \\
& c_{j}^{\varnothing}(P)=\left(\begin{array}{c}
f \\
j
\end{array}\right) .
\end{aligned}
$$

When $P \in \stackrel{\circ}{\mathrm{H}}_{n}$ (which is equivalent to $\min \left(n^{+}, n^{-}\right)>0$ ),

$$
\begin{aligned}
\Psi(P) & =\sum_{j=1}^{n}(-1)^{j-1}\left[\left(\begin{array}{l}
n \\
j
\end{array}\right)-\left(\begin{array}{c}
n^{+} \\
j
\end{array}\right)-\left(\begin{array}{c}
n^{-} \\
j
\end{array}\right)\right] \\
& =\left[1-(1-1)^{n}\right]-\left[1-(1-1)^{n^{+}}\right]-\left[1-(1-1)^{n^{-}}\right] \\
& =-1,
\end{aligned}
$$

with appropriate adjustment if $P \in \partial \mathrm{H}_{n}$ (equivalent to $\min \left(n^{+}, n^{-}\right)=0$ ). So, summation of $c_{j}(P)$ yields Theorem 2 for $h=1$ for all $P$ (not just 'almost all').

Lemma 3. Let the premises be the same as in Lemma 1, but now with $\min \left(n^{+}, n^{-}\right)>0$. Additionally, let $Z^{+} \subset \mathrm{F}^{+}$and $Z^{-} \subset F^{-}$be two zones adjacent in the sense that a facet $W$ of $Z^{+}$is a subset of $\mathrm{F}$ and is also a facet of $Z^{-}$. If $P \in W$ then $\Psi(P)=\Psi\left(Q^{+}\right)=\Psi\left(Q^{-}\right)$, where $Q^{+}$is any point in $Z^{+}$and $Q^{-}$is any point in $Z^{-}$.

If the case in which $n^{-}=0$ is also considered, then $Z^{-}$and $Q^{-}$are not defined, but $\Psi^{+}(P)=\Psi\left(Q^{+}\right)$still.

This lemma is illustrated for $h=2$ in Figure 4. The darkly-shaded region is the open zone $Z^{+}$, with the lightly-shaded region being $Z^{-}$. The 1 -flat $F$ is the line containing $A B$, whilst $W$ is the open line-segment (open 1-polytope) which separates the two shaded zones. The reference point $P \in W$. In Figure 3, the open shaded region is an example of $W$.

Proof of Lemma 3. Clearly $c_{j}\left(Q^{+}\right)=c_{j}^{+}(P)+c_{j}^{ \pm}(P)$ for any $j$. Therefore, $\Psi\left(Q^{+}\right)=$ $\Psi^{+}(P)+\Psi^{ \pm}(P)$ and, so, using $(9), \Psi\left(Q^{+}\right)=\Psi^{ \pm}(P)-\Psi^{\varnothing}(P)$. By a similar argument and 
(10), $\Psi\left(Q^{-}\right)=\Psi^{ \pm}(P)-\Psi^{\varnothing}(P)$. Both entities equal $\Psi(P)$, when $\min \left(n^{+}, n^{-}\right)>0$, because of (11). When $n^{-}=0$, the use of (9) combined with the obvious $\Psi^{ \pm}(P)=0$, establishes the stated results.

Proof of Theorem 2 for $P \in \partial \mathrm{H}_{n}$. We consider a facet of $\mathrm{H}_{n}$, called $\mathrm{T}$, and place an $(h-1)$ flat $\mathrm{F} \supset \mathrm{T}$. If we arbitrarily declare $\mathrm{F}^{+}$to be the half-space which intersects $\mathrm{H}_{n}$, then $n^{+}>0$ and $n^{-}=0$. Thus, from Lemma $1, \Psi(P)=0, P \in \mathrm{T}$. This argument can be applied to all facets, and so, to the whole boundary of $\mathrm{H}_{n}$.

Proof of Theorem 2 for almost all $P \in \stackrel{\circ}{\mathrm{H}}_{n}$. We prove that Theorem 2 is true for all $h$ and $n$ when $P$ lies in a 'restricted region' - namely, within any zone or in any zone's facet which is a subset of $\stackrel{\circ}{H}_{n}$. An induction is used.

Suppose that Theorem 2 is true in dimension $(h-1)$ for $P$ in the 'restricted region'. This means that when the action is taking place in dimension $h$, the theorem can be applied on the boundary of $\mathrm{H}_{n}$, in particular, within any $(h-1)$-polytope $\mathrm{T}$ which is a facet of $\mathrm{H}_{n}$. Thus, in the $h$-dimensional context, $\Psi^{\varnothing}(P)=(-1)^{h-1}$ for any $P$ lying in the 'restricted region' of $\mathrm{T}-\mathrm{a}$ region containing many facets of zones within $\mathrm{H}_{n}$.

If $P \in \mathrm{T}$ lies in a facet of such a zone (obviously a zone of $\mathrm{H}_{n}$ adjacent to $\mathrm{T}$ ), then Lemma 3 proves that $\Psi^{+}(P)=\Psi\left(Q^{+}\right)$for every point $Q^{+}$in that zone. Because Lemma 1 shows that $\Psi^{+}(P)=-\Psi^{\varnothing}(P)$, we have $\Psi\left(Q^{+}\right)=-\Psi^{\varnothing}(P)=(-1)^{h}$.

A clear consequence of Lemma 3 is that $\Psi(\cdot)$ is a constant, $\psi$ say, within the union of all the zones. If $\Psi(P)=\psi$ for $P$ in one zone, then the same is true in adjacent zones, their adjacent zones and so on. Lemma 3 also shows that $\Psi(\cdot)=\psi$ on all zone facets-and therefore within all of our 'restricted region' of $\mathrm{H}_{n}$. We readily see that the constant $\psi$ equals the $\Psi\left(Q^{+}\right)$in our previous paragraph; so $\psi=(-1)^{h}$.

The inductive argument is completed by noting that we have already proved the result for $h=1$.

Remark 4. In this proof of Theorem 2 we have gone further than required, as noted earlier when $h \leq 1$ (by replacing 'almost all' with 'all'), but also when $h \geq 2$. In this latter case the set of positions for $P \in \stackrel{\circ}{\mathrm{H}}$ not covered by our proof, namely those positions which lie on a zonal $i$-face, where $0 \leq i \leq(h-2)$, is of dimension $(h-2)$, two dimensions lower than the space where the action takes place.

For some of the 0 -faces, we have an added result.

Lemma 4. Suppose that $P \in \stackrel{\circ}{\mathrm{H}}_{n}$ is a zonal 0-face that coincides with a point $P_{i}$, which without loss of generality we can call $P_{n}$ (because points can be relabelled to suit our needs). Define $c_{j}(P, m)$ as the number of $P$-covering $j$-hulls taken only from $\left\{P_{1}, P_{2}, \ldots, P_{m}\right\}$, where $1 \leq m \leq n$, and let $\Psi(P, m):=\sum_{j=1}^{m}(-1)^{j-1} c_{j}(P, m)$. Then, for $n \geq 2$,

$$
\Psi\left(P_{n}\right) \equiv \Psi\left(P_{n}, n\right)=\Psi\left(P_{n}, n-1\right) .
$$

Proof. We can write

$$
c_{j}\left(P_{n}\right) \equiv c_{j}\left(P_{n}, n\right)=c_{j}\left(P_{n}, n-1\right)+\left(\begin{array}{c}
n-1 \\
j-1
\end{array}\right),
$$


where the second term captures the idea that all $(j-1)$-subsets from $\left\{P_{1}, P_{2}, \ldots, P_{n-1}\right\}$, when augmented with $P_{n}$, have convex hull covering $P_{n}$. Then

$$
\begin{aligned}
\Psi\left(P_{n}\right) \equiv \Psi\left(P_{n}, n\right) & =\sum_{j=1}^{n}(-1)^{j-1}\left[c_{j}\left(P_{n}, n-1\right)+\left(\begin{array}{c}
n-1 \\
j-1
\end{array}\right)\right] \\
& =\Psi\left(P_{n}, n-1\right)+(-1)^{n-1} c_{n}\left(P_{n}, n-1\right)+(1-1)^{n-1} \\
& =\Psi\left(P_{n}, n-1\right) \quad \text { if } n \geq 2,
\end{aligned}
$$

since $c_{n}\left(P_{n}, n-1\right)=0$.

\section{Proof of the topological result for all positions of $P$ when $h=2$}

We conjecture that the result in Theorem 2 is valid for all $P \in \stackrel{\circ}{\mathrm{H}}$, not just 'almost all', for any dimension $h>1$. We conclude the paper by establishing this conjecture for $h=2$, using Lemma 4 combined with other computations.

Theorem 3. When $h=2$ (implying $n \geq 3$ ), $\Psi(P)=(-1)^{2}=1$ for all $P \in \stackrel{\circ}{\mathrm{H}}$.

Proof. The positions of $P \in \stackrel{\circ}{\mathrm{H}}_{n}$ not included in Theorem 2 are at the corners (0-faces) of zones. Most of these corners are located in the interior of some 2-hull, whilst the remaining few are coincident with one of the points $P_{i} \in \stackrel{\circ}{\mathrm{H}}_{n}$ (for example, point $G$ in Figure 2(b), a point not in the interior of a 2-hull).

Suppose $P \in \stackrel{\circ}{\mathrm{H}}_{n}$ lies in the interior of some 2-hull, which without loss of generality can be the line-segment $P_{n-1} P_{n}$. Let $\mathrm{F}$ be the flat which covers this line-segment, with $n^{+}$and $n^{-}$being defined relative to $\mathrm{F}$. We introduce the notation $f_{j}(\mathrm{~F}, m)$ as the number of $\mathbf{F}$ intersecting $j$-hulls taken only from $\left\{P_{1}, P_{2}, \ldots, P_{m}\right\}$, where $1 \leq m \leq n$. Then, we can write $c_{1}(P)=c_{1}(P, n-2)$ and, when $j \geq 2$,

$$
\begin{aligned}
c_{j}(P) \equiv c_{j}(P, n)= & c_{j}(P, n-2)+2 c_{j-1}(P, n-2) \\
& +\left[f_{j-1}(\mathrm{~F}, n-2)-c_{j-1}(P, n-2)\right]+\left(\begin{array}{c}
n-2 \\
j-2
\end{array}\right) .
\end{aligned}
$$

The second term captures the fact that any $P$-covering $(j-1)$-hull taken only from $\left\{P_{1}, P_{2}, \ldots, P_{n-2}\right\}$ can be augmented with either $P_{n-1}$ or $P_{n}$ to give a $P$-covering $j$-hull. The term in square-brackets counts the number of $(j-1)$-hulls taken only from $\left\{P_{1}, P_{2}, \ldots, P_{n-2}\right\}$ which intersect $\mathrm{F}$ but do not cover $P$-and each of these makes a $P$-covering $j$-hull when augmented with the appropriate point $P_{n-1}$ or $P_{n}$. The last term captures the idea that all $(j-2)$-subsets from $\left\{P_{1}, P_{2}, \ldots, P_{n-2}\right\}$, when augmented with $P_{n-1}$ and $P_{n}$, have convex hull which covers $P$. Thus, using Lemma 2 to evaluate $f_{j-1}(\mathrm{~F}, n-2)$, we have

$$
\begin{aligned}
c_{j}(P) \equiv c_{j}(P, n)= & c_{j}(P, n-2)+c_{j-1}(P, n-2) \\
& +\left(\begin{array}{c}
n-2 \\
j-1
\end{array}\right)-\left(\begin{array}{c}
n^{+} \\
j-1
\end{array}\right)-\left(\begin{array}{c}
n^{-} \\
j-1
\end{array}\right)+\left(\begin{array}{c}
n-2 \\
j-2
\end{array}\right) .
\end{aligned}
$$


So, when the line-segment $P_{n-1} P_{n} \not \subset \partial \mathrm{H}_{n}$ (implying that $n \geq 4$ and $\min \left(n^{+}, n^{-}\right) \geq 1$ ),

$$
\begin{aligned}
\Psi(P) \equiv & \Psi(P, n) \\
= & \Psi(P, n-2)-\Psi(P, n-2)+\left[(1-1)^{n-2}-1\right]-\left[(1-1)^{n^{+}}-1\right] \\
& -\left[(1-1)^{n^{-}}-1\right]-(1-1)^{n-2} \\
= & 1 .
\end{aligned}
$$

If the segment $P_{n-1} P_{n} \subset \partial \mathrm{H}_{n}$, whereby $n \geq 3, n^{-}=0$, and $n^{+} \geq 1$, then a minor adjustment to the calculation above shows that $\Psi(P)=0$. Therefore, Theorem 3 is true when $P$ is located at a zonal corner which lies in the interior of a 2-hull. This leaves only some remaining positions for $P \in \stackrel{\circ}{\mathrm{H}}_{n}$, where $P=P_{i}$, for some $i$. However, we note that there are no such positions in $\mathrm{H}_{N}$, where, $N:=\min \left(m: \operatorname{dim}\left(\mathrm{H}_{m}\right)=2\right) \geq 3$. So, $\Psi(P, N)=1$ for all $P \in \stackrel{\circ}{\mathrm{H}}_{N}$ and, by induction the theorem is proved-using Lemma 4 to cater for any positions of $P$ not covered by Theorem 2 and (14) - firstly for $N+1$ points, then $N+2$ points and so on, until the theorem is proved for $n$ points.

\section{Appendix A. Other identities involving volume moments}

Some results for higher moments emerge from our almost-sure identity (4). For example, consider $n=d+2$, where Buchta's result is $\mathrm{E}\left(V_{d+2}\right)=\frac{1}{2}(d+2) \mathrm{E}\left(V_{d+1}\right)$. This result strengthens to

$$
V_{d+2}=\frac{1}{2}(d+2) \bar{V}_{d+1}^{(d+2)}=\frac{1}{2}\left[V_{(1)}^{(d+2)}+V_{(2)}^{(d+2)}+\cdots+V_{(d+2)}^{(d+2)}\right],
$$

where $V_{(j)}^{(n)}$ is the volume of the convex hull of all the $n$ points except $P_{j}$. A consequence of this breakdown of $V_{d+2}$ into the sum of $(d+2)$ exchangeable entities is a new relationship,

$$
\operatorname{var}\left(V_{d+2}\right)=\frac{1}{4}(d+2)\left[\operatorname{var}\left(V_{d+1}\right)+(d+1) \operatorname{cov}\left(V_{(1)}^{(d+2)} V_{(2)}^{(d+2)}\right)\right] .
$$

We also note other recursion formulae found from (2) by strategic 'manipulation' of the $\mathrm{E} V_{n-j}$ terms on its right-hand side when the subscript has the right parity, that is, when $(n-j-d) \geq 2$ and even. Various such forms (details of proof omitted) follow. For $(n-d) \geq 2$ and even,

$$
\begin{aligned}
\mathrm{E}\left(V_{n}\right)= & \sum_{j=1}^{n-d-1}(-1)^{j-1}\left(\begin{array}{c}
n / 2 \\
j
\end{array}\right) \mathrm{E}\left(V_{n-j}\right), \\
= & \left.\left(\begin{array}{l}
n \\
d
\end{array}\right) /\left(\begin{array}{c}
(n+d) / 2 \\
d
\end{array}\right)\right) \\
& \times \sum_{j=1}^{(n-d) / 2}(-1)^{j-1}\left(\left(\begin{array}{c}
d+j \\
j
\end{array}\right)\left(\begin{array}{c}
(n+d) / 2 \\
d+j
\end{array}\right) /\left(\begin{array}{c}
n-j \\
d
\end{array}\right)\right) \mathrm{E}\left(V_{n-j}\right) \\
= & \sum_{j=1}^{n-d-1}(-1)^{j-1}\left(\begin{array}{c}
n \\
j
\end{array}\right) \frac{\mathrm{E}\left(V_{n-j}\right)}{j+1} .
\end{aligned}
$$

The last of these three forms can also be derived from (8). 


\section{References}

[1] Affentranger, F. (1988). Generalization of a formula of C. Buchta about the convex hull of random points. Elem. Math. 43, 39-45, 151-152.

[2] Baddeley, A. J. AND Møller, J. (1989). Nearest-neighbour Markov point processes and random sets. Internat. Statist. Rev. 57, 89-121.

[3] Baddeley, A. J. and van Lieshout, M. N. M. (1995). Area-interaction point processes. Ann. Inst. Statist. Math. 47, 601-619.

[4] Badertscher, E. (1989). An explicit formula about the convex hull of random points. Elem. Math. 44, 104-106.

[5] Buchta, C. (1986). On a conjecture of R. E. Miles about the convex hull of random points. Monatsh. Math. 102, 91-102.

[6] Buchta, C. (1990). Distribution-independent properties of the convex hull of random points. J. Theoret. Prob. 3, 387-393.

[7] Grünbaum, B. (1967). Convex Polytopes. John Wiley, London.

[8] Miles, R. E. (1971). Isotropic random simplices. Adv. Appl. Prob. 3, 353-382.

[9] Strauss, D. J. (1975). A model for clustering. Biometrika 63, 467-475. 